\section{Health of the Nation Outcome Scales for People with Learning Disabilities (HoNOS-LD)}

\author{
Glossary for HoNOS-LD score sheet ${ }^{\dagger}$
}

\author{
ASHOK ROY, HELEN MATTHEWS, PAUL CLIFFORD, VANESSA FOWLER \\ and DAVID M. MARTIN
}

Summary rating instructions:

(a) Complete the front sheet including ICD-10 diagnoses and subjective rating.

(b) Rate each in order from item 1 to 18.

(c) Do not include information rated in an earlier item.

(d) Rate the person over the previous 4 weeks.

(e) Rate the most severe problem that has occurred during the period rated.

(f) All items follow the five-point rating format similar to other HoNOS instruments:

$0=$ no problem during the period rated; $1=$ mild problem;

$2=$ moderate problem;

$3=$ severe problem;

$4=$ very severe problem.

\section{Rate 9 if unknown}

\section{Behavioural problems (directed at others)}

Include behaviour that is directed to other persons. Do not include behaviour that is directed towards self (Scale 2) or primarily at property or other behaviours (Scale 3). Rate risk as it is currently perceived.

$0=$ No behavioural problems directed to others during the period rated.

$1=$ Irritable, quarrelsome, occasional verbal abuse.

$2=$ Frequent verbal abuse, verbal threats, occasional aggressive gestures, pushing or pestering (harassment).

$3=$ Risk, or occurrence of, physical aggression resulting in injury to others requiring simple first aid, or requiring close monitoring for prevention.

$4=$ Risk, or occurrence of, physical aggression producing injury to others serious

†See pp. 6I-66, this issue. enough to need casualty treatment and requiring constant supervision or physical intervention for prevention (e.g. restraint, medication or removal).

\section{Behavioural problems directed towards self (self-injury)}

Include all forms of self-injurious behaviour. Do not include behaviour directed towards others (Scale 1), or behaviour primarily directed at property, or other behaviours (Scale 3).

$0=$ No self-injurious behaviour during the period rated.

$1=$ Occasional self-injurious behaviour (e.g. face-tapping); occasional fleeting thoughts of suicide.

$2=$ Frequent self-injurious behaviour not resulting in tissue damage (e.g. redness, soreness, wrist-scratching)

$3=$ Risk or occurrence of self-injurious behaviour resulting in reversible tissue damage and no loss of function (e.g. cuts, bruises, hair loss).

$4=$ Risk or occurrence of self-injurious behaviour resulting in irreversible tissue damage and permanent loss of functions (e.g. limb contractures, impairment of vision, permanent facial scarring) or attempted suicide.

\section{Other mental and behavioural problems}

This is a global rating to include behavioural problems not described in Scales 1 or 2. Do not include behaviour directed towards others (Scale 1), or self-injurious behaviour (Scale 2). Rate the most prominent behaviours present. Include: A, behaviour destructive to property; $\mathrm{B}$, problems with personal behaviours, for example, spitting, smearing, eating rubbish, self-induced vomiting, continuous eating or drinking, hoarding rubbish, inappropriate sexual behaviour; C, rocking, stereotyped and ritualistic behaviour; D, anxiety, phobias, obsessive or compulsive behaviour; E, others.

$0=$ No behavioural problem $(\mathrm{s})$ during the period rated.

$1=$ Occasional behavioural problem(s) that are out of the ordinary or socially unacceptable.

$2=$ Behaviour(s) sufficiently frequent and severe to produce some disruption of and impact on own or other people's functioning.

$3=$ Behaviour(s) sufficiently frequent and severe to produce significant disruption and impact on own or other people's functioning, requiring close monitoring for prevention.

$4=$ Constant, severe problem behaviour(s) producing major disruption of and impact on functioning requiring constant supervision or physical intervention for prevention.

\section{Attention and concentration}

Include problems that may arise from underactivity, overactive behaviour, restlessness, fidgeting or inattention, hyperkinesis or arising from drugs.

$0=$ Can sustain attention and concentration in activities/programmes independently during the period rated.

$1=$ Can sustain attention and concentration in activities/programmes with occasional prompting and supervision.

$2=$ Can sustain attention and concentration in activities/programmes with regular prompting and supervision.

$3=$ Can sustain attention and concentration in activities/programmes briefly with constant prompting and supervision.

$4=$ Cannot participate in activities and programmes even with constant prompting and supervision.

\section{Memory and orientation}

Include recent memory loss and worsening of orientation for time, place and person in addition to previous difficulties.

$0=$ Can reliably find their way around familiar surroundings and relate to familiar people.

$1=$ Mostly familiar with environment/ person, but with some difficulty in finding their way.

$2=$ Can relate to environment/person with occasional support and supervision. 
$3=$ Can relate to environment/person with regular support and supervision.

$4=$ Not apparently able to recognise or relate to people and environments.

\section{Communication (problems with understanding)}

Include all types of responses to verbal, gestural and signed communication, supported if necessary with environmental cues.

$0=$ Able to understand first language (mother tongue) about personal needs and experience during the period rated.

$1=$ Able to understand groups of words/ short phrases/signed communication about most needs.

$2=$ Able to understand some signs, gestures and single words about basic needs and simple commands (food, drink, come, go, sit, etc.).

$3=$ Able to acknowledge and recognise attempts at communication with little specific understanding (pattern of response is not determined by nature of communication).

$4=$ No apparent understanding or response to communication.

\section{Communication (problems with expression)}

Include all attempts to make needs known and communicate with others (words, gestures, signs). Rate behaviour under Scales 1, 2 and 3.

$0=$ Able to express needs and experience during the period rated.

$1=$ Able to express needs to familiar people.

$2=$ Able to express basic needs only (food, drink, toilet, etc.).

$3=$ Able to express presence of needs, but cannot specify (e.g. cries or screams when hungry, thirsty or uncomfortable).

$4=$ Unable to express need or presence of need.

\section{Problems associated with hallucinations and delusions}

Include hallucinations and delusions irrespective of diagnosis. Include all manifestations suggestive of hallucinations and delusions (responding to abnormal experiences, e.g. invisible voices when alone).
$0=$ No evidence of hallucinations or delusions during period rated.

$1=$ Occasional odd or eccentric beliefs or behaviours suggestive of hallucinations or delusions.

$2=$ Manifestations of hallucinations or delusions with some distress or disturbance.

3= Manifestations of hallucinations or delusions with significant distress or disturbance.

$4=$ Mental state and behaviour are seriously and adversely affected by hallucinations or delusions with severe distress or disturbance.

\section{Problems associated with mood changes}

Include problems associated with low mood states, elated mood states, mixed moods and mood swings (alternating between unhappiness, weeping and withdrawal on one hand and excitability and irritability on the other).

$0=$ No evidence of mood change during period rated.

$1=$ Mood present but with little impact (e.g. gloom).

$2=$ Mood change producing significant impact on self or others (e.g. weeping spells, decrease in skills, withdrawal and loss of interest).

$3=$ Mood change producing major impact on self or others (e.g. severe apathy and unresponsiveness, severe agitation and restlessness).

$4=$ Depression, hypomania or $\operatorname{mood}$ swings producing severe impact on self and others (e.g. severe weight loss from anorexia or overactivity, agitation too severe to allow time to be engaged in meaningful activity).

\section{Problems with sleeping}

Do not rate intensity of behaviour disturbance - this should be included in Scale 3 . Include daytime drowsiness, duration of sleep, frequency of waking and diurnal variation of sleep pattern.

$0=$ No problem during the period rated.

$1=$ Occasional mild sleep disturbance with occasional waking.

$2=$ Moderate sleep disturbance with frequent waking, or some daytime drowsiness.
$3=$ Severe sleep disturbance or marked daytime drowsiness (e.g. restlessness/ overactivity/waking early) on some nights.

$4=$ Very severe sleep disturbance with disturbed behaviour (e.g. restlessness/ overactivity/waking early most nights).

\section{Problems with eating and drinking}

Include both increase and decrease in weight. Do not rate pica - which should be rated in Scale 3. This scale does not include problems experienced by people who cannot feed themselves (e.g. people with severe physical disability).

$0=$ No problem with appetite during the period rated.

$1=$ Slight alteration to appetite.

$2=$ Severe alteration in appetite with no significant weight change.

$3=$ Severe disturbance with some weight change during the period rated.

$4=$ Very severe disturbance with significant weight change during the period rated.

\section{Physical problems}

Include illnesses from any cause that adversely affects mobility, self-care, vision and hearing (e.g. dementia, thyroid dysfunction, tremor affecting dexterity). Do not include relatively stable physical disability (e.g. cerebral palsy, hemiplegia). Behavioural disorders caused by physical problems should be rated under Scales 1 , 2 and 3 (e.g. constipation producing aggression).

$0=$ No increased incapacity due to physical problems during the period rated.

$1=$ Mildly increased incapacity, for example, viral illness, sprained wrist.

$2=$ Significant incapacity requiring prompting and supervision.

$3=$ Severe incapacity requiring some assistance with basic needs.

$4=$ Total incapacity requiring assistance for most basic needs such as eating and drinking, toileting (fully dependent).

\section{Seizures}

Include all types of fits (partial, focal, generalised, mixed, etc.) to rate the shortterm effect on the individual's daily life. Rate the effects of the fits. Do not include 
behavioural problems caused by, or associated with, fits (use Scales 1, 2 and 3).

$0=$ No increased incapacity due to physical problems during the period rated.

$1=$ Occasional seizures with minimal immediate impact on daily activities (e.g. resumes after seizures).

$2=$ Seizures of sufficient frequency or severity to produce a significant immediate impact on daily activities (e.g. resumes activity after a few hours).

$3=$ Seizures of sufficient frequency or severity producing a severe immediate impact on daily activities requiring simple first aid for injuries etc. (e.g. resumes activities next day).

$4=$ Frequent poorly controlled seizures (may be accompanied by episodes of status epilepticus) requiring urgent clinical attention.

\section{Activities of daily living at home}

Include such skills as cooking, cleaning and other household tasks. Do not rate problems with daily living outside the home (Scale 15). Do not rate problems with selfcare (Scale 16). Rate what is seen regardless of cause, for example, disability, motivation etc. Rate performance not potential. Rate the current level achieved with the existing support.

$0=$ Performs or contributes towards activities of daily living at home.

$1=$ Some limitations in performing or contributing towards household tasks.

$2=$ Significant limitations in performing or contributing towards household tasks (e.g. failure to wash or tidy up, difficulty in preparing meals).

$3=$ Major limitations in performing or contributing towards household tasks (e.g. home neglected, dirty, untidy; no domestic routine).

$4=$ Gross neglect or danger resulting from no apparent contribution to daily living activities.

\section{I5. Activities of daily living outside the home}

Include skills such as budgeting, shopping, mobility and the use of transport, etc. Do not include problems with activities of daily living at home (Scale 14). Do not rate

ASHOK ROY, FRCPsych, Brooklands, Marston Green, Birmingham; HELEN MATTHEWS, MRCPsych, St David's, Carmarthen; PAULCLIFFORD, DClinPsy, British Psychological Society; VANESSA FOWLER, MSc, Galahad Substance Misuse Solutions Ltd, Oxted, Surrey; DAVID M. MARTIN, BA, Brooklands, Marston Green, Birmingham, UK

Correspondence: Dr A. Roy, North Warwickshire NHS Trust, Brooklands, Coleshill Road, Marston Green, Birmingham B37 7HL, UK

(First received 29 March 2000, final revision 19 September 2000, accepted 19 September 2000)

problems with self-care (Scale 16). Rate the current level with the existing support.

$0=$ Regular use of facilities and public amenities (e.g. shopping).

$1=$ Some limitation in activity (e.g. difficulty with the use of public amenities or transport).

$2=$ Significant limitations of activity relating to any one of: shopping, use of transport, public amenities.

$3=$ Major restrictions in activity relating to more than any one of: shopping, use of transport, public amenities.

$4=$ Severe restrictions in the use of shops, transport, facilities, etc.

\section{Level of self-care}

Rate the overall level of functioning in activities of self-care such as eating, washing, dressing and toileting. Rate the current level achieved with the existing support. Rate appearance not motivation.

$0=$ Appearance and personal hygiene maintained.

$1=$ Some deficits in personal appearance, personal hygiene or attention to health (e.g. poor grooming)

$2=$ Significant deficits in personal appearance, personal hygiene or attention to health causing a problem with social acceptability, but not sufficient to pose a health risk (e.g. body odour, unkempt hair or nails).

$3=$ Major deficits in personal appearance, personal hygiene or attention to health posing a health risk (e.g. skin rashes, gum infection, not fully dressed).

$4=$ Gross self-neglect with severe difficulties relating to appearance, hygiene and diet posing a major health risk (e.g. pressure sores).

\section{Problems with relationships}

Include effects of problems with relationships with family, friends and carers (in residential and day/leisure settings). Measure what is occurring regardless of cause, for example, somebody who is known to have good relationships may still display problems.

$0=$ Positive and frequent contact with family or friend or carers.

$1=$ Generally positive relationships, but some strain or limitations in contact.

$2=$ Some positive relationships, but current disruptions of contact or worsening of relationships.

$3=$ Difficulties in relationships with risk of breakdown or infrequent contact.

$4=$ Significant relationships broken down with no current contact.

\section{Occupation and activities}

Rate the overall level of problems with quality of daytime environment. Take account of frequency and appropriateness of, and engagement with, daytime activities. Consider factors such as lack of qualified staff, equipment and appropriateness with regard to age and clinical condition. Do not rate problems with self-care (Scale 16).

$0=$ Fully engaged with acceptable range of activities.

$1=$ Uses reasonable range of activities, but some limitation of access or appropriateness.

$2=$ Uses limited range of activities, limited availability or appropriateness.

$3=$ Attends daytime activity irregularly.

$4=$ No engagement with daytime activity. 


\section{APPENDIX}

HoNOS-LD score sheet

Scale 0-4

I. Behavioural problems

Rate 9 if not known (directed at others)

2. Behavioural problems directed towards self (self-injury)

3. Other mental and behavioural problems (specify problem, A, B, C, D or $\mathrm{E})$
4. Attention and concentration

5. Memory and orientation

6. Communication (problems with understanding)

7. Communication (problems with expression)

8. Problems associated with hallucinations and delusions

9. Problems associated with mood changes

10. Problems with sleeping
II. Problems with eating and drinking

12. Physical problems

13. Seizures

14. Activities of daily living at home

15. Activities of daily living outside the home $\square$

16. Level of self-care

17. Problems with relationships

18 Occupation and activities

Total score

Subjective score 